\title{
Fracture mechanics of laser sintered cracked Polyamide for a new method to induce cracks by additive manufacturing
}

\author{
T.Brugo ${ }^{a}$, R.Palazzettib, *, S. Ciric-Kostic ${ }^{c}$, X.T. Yan ${ }^{\text {b }}$ G.Minak ${ }^{\mathrm{a}}$ and \\ A.Zucchellia \\ aUniversity of Bologna, DIN department, Viale del Risorgimento 2, 40136, \\ Bologna, Italy. Email: tommasomaria.brugo@unibo.it \\ bUniversity of Strathclyde, DMEM department, 75 Montrose Street, G1 1XJ, \\ Glasgow, UK. Email: roberto.palazzetti@strath.ac.uk \\ cUniversity of Kragujevac, Faculty of Mechanical and Civil Engineering in \\ Kraljevo, Dositejeva 19, 36000, Kraljevo, Serbia. Email: \\ cirickostic.s@mfkv.kg.ac.rs \\ * Corresponding author
}

\begin{abstract}
This paper presents an experimental investigation on specimens manufactured by Selective Laser Sintering (SLS), with the purposes of giving designers advice when designing 3D printed parts, and laying the basis for a step forward in the field of fracture mechanics of 3D complex parts.

The aim is to investigate the effect of building direction in Polyamide (PA) 3D printed samples and to assess whether a crack can be initiated directly from the sintering process for fracture mechanics study purposes.

Six different configurations of Mode I Compact Tension (CT) specimens were manufactured and tested; the experiments were monitored by Digital Image Correlation (DIC) and fractured surfaces were analyzed using microscopy. Results showed that samples with better mechanical performance are those in which all the layers contain a portion of the crack. On the other hand, those with layers parallel to the crack plan offer a preferential pathway for the crack to propagate. DIC and fractography investigations showed that, under certain conditions, small-radius geometries, or too-close surfaces may glue depending on printer resolution. Experiments also showed that SLS is capable of printing
\end{abstract}


specimens with internal cracks that can be used to study fracture mechanics of complex parts or parts with internal cracks.

\section{Keywords}

Selective Laser Sintering, Fracture Mechanics, Digital Control Imaging, Additive Manufacturing

\section{Introduction}

Selective Laser Sintering (SLS) is one of the most popular additive manufacturing (AM) techniques: it uses a laser beam to sinter powder to build objects bottomup, layer-by-layer [1]. SLS can be applied to a large range of materials such as metals, ceramic, wax or polymers, and has nowadays spread to a large range of products, from aerospace to sports-car components [2].

SLS presents several advantages if compared with traditional manufacturing techniques for plastics, especially for low or medium size batches, mainly due to the combination of design freedom given by the AM technique, and the large range of materials that can be processed [3].

Homogeneity and isotropy of the SLS products are big issues, especially when designing structural components. Chooke at al. [4] performed a rigorous analysis of the Young's modulus, ultimate tensile strength, and strain-to-failure of a glassfilled nylon PA12 composite called DuraForm $®$, demonstrating the variability of the material properties throughout the built volume and between builds. Berti et al. [5] also tensile tested specimens built with different orientations, at two different temperatures. They found that the most important anisotropy is located between the vertical direction and the horizontal plane, but near the upper operative temperature the mechanical difference between specimens built with longitudinal axis parallel to the two horizontal directions becomes important. They also proposed a model where it is hypothesized that the layerby-layer construction is only marginally responsible for the anisotropic behavior of the material. Zhang et al. [6] 3D printed alumina/glass composites, and investigated the effect of sample orientation within the building chamber on microstructure, porosity and mechanical properties of the final products. They showed that sintered samples orientated parallel to print head direction exhibit 
significantly higher fracture toughness than those printed in the other two orientations. The same authors showed that mechanical properties of the samples significantly improve by adding lanthanum-alumino-silicate glass into sintered porous alumina preforms, and tend to be no longer dependent on building direction.

Similar results have been found by Shi et al [7], who post-treated polycarbonate (PC) specimens infiltrated with epoxy resin, showing great enhancement of mechanical properties. At the same time, they found that the average dimensional error of PC SLS specimens is not satisfactory, ranging from 6.67 to 13.6 per cent. Cain et al. [8] performed similar analysis on $\mathrm{Ti}_{6} \mathrm{Al}_{4} \mathrm{~V}$ Selective Laser Melted (SLM) samples, and found that building direction strongly affect the fracture toughness and the fatigue crack growth rate of the samples due to anisotropic residual stress distribution. Furthermore, they observed that building direction has much less influence on the uniaxial tensile properties of the material. Their results were confirmed by Vrancken et al. [9], who showed that the residual stress inside samples is mainly oriented along the building direction, significantly affecting the fracture behavior during fracture toughness tests.

In the present work, three point bending tests were performed on non-fractured samples, built at different directions, in order to assess the isotropy of the printed material. Polyamide (PA) specimens manufactured by SLS; three different configurations were manufactured with the purpose of evaluating the effect of building direction on Mode I fracture mechanic properties of the material. Similar studies on $\mathrm{PA}-\mathrm{Al}_{2} \mathrm{O}_{3}$ composites have been presented by $[5,6]$, mainly focused on tensile or bending properties. The novelty of this work lays in its focus on the fracture mechanics properties of PA for SLS, and on the investigation on the capability of AM processes to build 3D artificial cracks, otherwise impossible to create by classic methods. Mode I Compact Tension (CT) specimens were manufactured and tested, and the results are evaluated by using stress-strain curves, fractography images and Digital Image Correlation (DIC). The DIC technique [10] allows full field contactless measurements of displacement and strain of the specimen being tested, and was found to be particularly useful for the purpose of the present study. Specifically in the field of 
fracture mechanics, it has been used to obtain fracture toughness by measuring the displacement field at the tip of the crack. In particular, McNeill et al. [11] performed experiments on Compact and Three Point Bending (3PB) specimens and compared results between DIC and analytical data, suggesting a methodology to use the DIC data do determine the fracture toughness $\mathrm{KI} C$ of specimens.

In the present work, the DIC technique was used by full strain field analysis with the double purpose of monitoring crack tip path and irregular behavior.

\section{Materials and methods}

Eighteen CT specimens were manufactured in a EOS Formiga P100 SLS machine using fine Polyamide PA 2200 powder purchased from Plastic Design \& Service (Sweden, www.pds.se), which main properties are listed in Table 1. The lasersintered specimens were produced from recycled powder mixed with $50 \%$ of new powder.

\begin{tabular}{ll}
\hline \multicolumn{1}{c}{ Property } & \multicolumn{1}{c}{ Value } \\
Sintered Density & $0.9-0.95 \mathrm{Kg} / \mathrm{dm}^{3}$ \\
Flexural Young's Modulus & $1.5 \mathrm{GPa}$ \\
Flexural Strength & $58 \mathrm{MPa}$ \\
Elongation at break & $15 \%$ \\
\hline Table 1: PA properties. Source: Plastic Design \& Service
\end{tabular}

Specimens were manufactured and tested following the guidelines provided in the International Standard ISO 13586 [12]: characteristic dimensions $B$ and $W$ were 16 and $32 \mathrm{~mm}$ respectively (see Figure 1). Characteristic dimensions of the samples have been chosen to comply with the requirement of the standard, in particular the $B, a, W-a>2.5\left(K_{Q} / \sigma_{y}\right)$ constraint $\left(\mathrm{K}_{\mathrm{Q}}\right.$ is the conditional or trial $\mathrm{K}_{\mathrm{Ic}}$ value, and $\sigma_{\mathrm{y}}$ is the yield stress of the material for the temperature and loading rate of the test). 


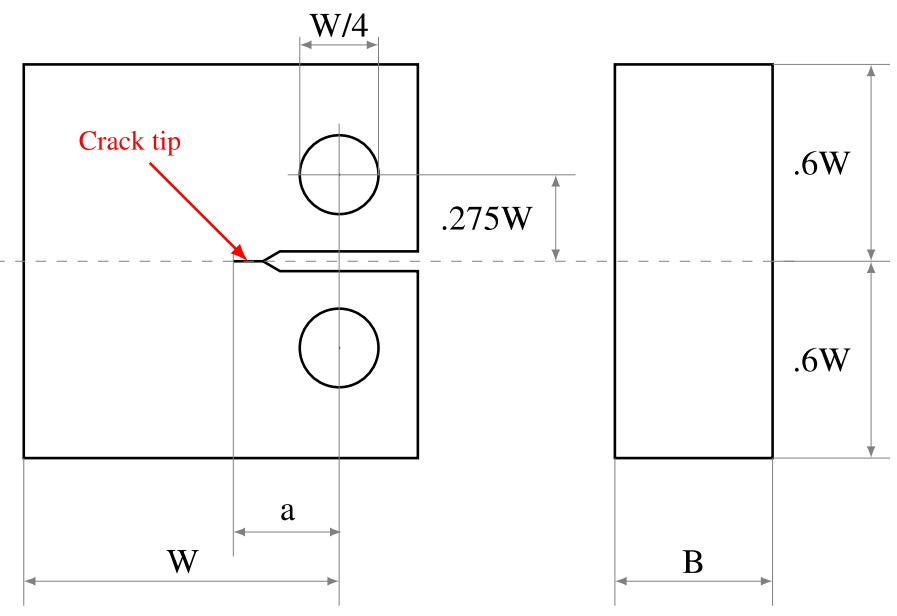

The samples were manufactured using a $30 \mathrm{~W}$ power RF-excited $\mathrm{CO}_{2}$ laser with $10.6 \mu \mathrm{m}$ wavelength and $254 \mu \mathrm{m}$ diameter laser beam. The powder was preheated to $172^{\circ} \mathrm{C}$, the laser beam power was $21 \mathrm{~W}$, the laser scan speed was $2500 \mathrm{~mm} / \mathrm{s}$ and the thickness of individual PA2200 layer was $100 \mu \mathrm{m}$. The laser power was chosen in order to obtain the highest density without compromising the polymer integrity: as shown by Hitt et al. [13], high laser power leads to more effective sintering but, over a certain value, the polymer degrades. Six different configurations were manufactured changing (i) the way the crack tip was realized, and (ii) the sample orientation in the printing chamber. In particular:

- specimens were built in three different orientations, designated XY (all the layers contain a segment of the crack), XZ (layers perpendicular to the crack plane) and YZ (layers parallel to the crack plane), as shown in Figure 2; 


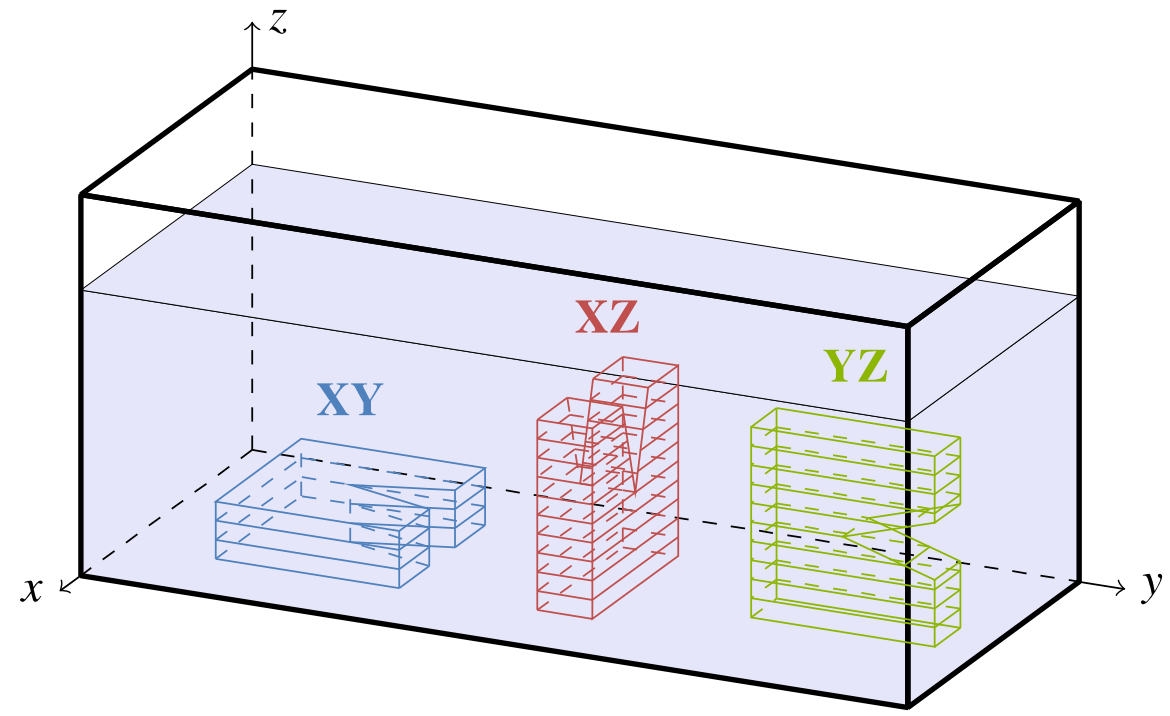

(a) YZMAN fracture surfaces

(b) YZsLs fracture surfaces

- cracks were initiated in two different ways: nine specimens had the crack manufactured during the sintering, while the remaining samples had the crack initiated after the manufacturing. The two crack tips identify two configurations, designated MAN (for specimens which crack is initiated after the manufacturing) and SLS (for specimens with the crack manufactured). The $S L S$ specimens had a $0.3 \mathrm{~mm}$ thick crack included in the 3D model, which allowed the AM process to manufacture specimens already cracked. The $M A N$ specimens were cracked by pressing a razor blade into the notch, as suggested by [14]: in this way a sharp crack is obtained in a fraction of the time required to grow a fatigue crack, and the measured toughness is not adversely affected if the notching is done properly [15]. Crack tips are shown in Figure 3. 


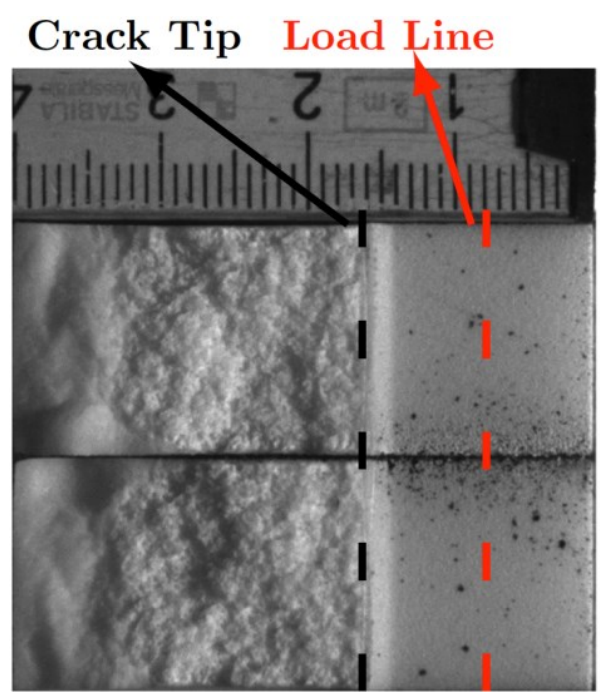

(a) MAN specimen: crack length and stress concentration

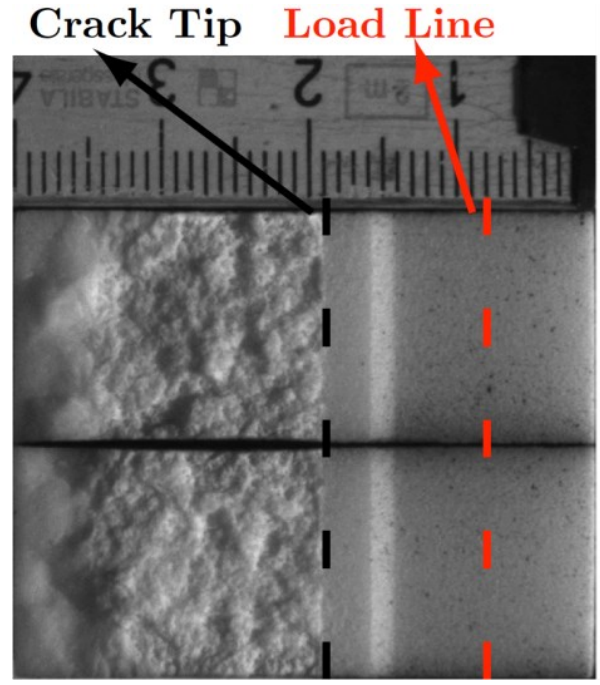

b) SLS specimen: crack length and stress concentration

Due to different crack manufacturing processes, MAN and SLS crack lengths were 8 and $10.7 \mathrm{~mm}$ long, respectively; our experimental equipment did not allow to make a $10.7 \mathrm{~mm}$ deep crack as that of SLS samples. This makes the force-displacement curves of the two configurations not directly comparable with each other, but still allows comparison of fracture mechanics properties (such as $\mathrm{K}_{\mathrm{IC}}$ ) between configurations. Fracture toughness is an intrinsic property of the material and, therefore, its calculation does not depend on the initial crack length.

Three specimens for each configuration have been manufactured and tested. Digital Image Correlation was used to measure strain field around the crack tip during the tests. The testing machine was an INSTRON 8032 equipped with a 25 $\mathrm{kN}$ load cell, running with a cross head speed of $3 \mathrm{~mm} / \mathrm{min}$; load-displacement curves were obtained recording force and crack opening displacement (COD) 50 times per second.

In order to assess that the material properties are not affected by the building direction, Young's modulus (E) and flexural yield stress $\left(\sigma_{\mathrm{y}}\right)$ were measured by three point bending tests on un-notched specimens, and the results are shown in Table 2. 


\begin{tabular}{rcccc}
\hline \multirow{2}{*}{ Configuration } & \multicolumn{2}{c}{$\sigma_{\mathrm{y}}$ (MPa) } & \multicolumn{2}{c}{ E (GPa) } \\
& $\mu$ & $\sigma$ & $\mu$ & $\sigma$ \\
$\mathrm{XY}$ & 54.0 & 1.4 & 1.22 & 0.02 \\
$\mathrm{YZ}$ & 53.5 & 0.7 & 1.25 & 0.03 \\
$\mathrm{XZ}$ & 55.0 & 1.4 & 1.26 & 0.03
\end{tabular}

Table 2: Three point bending tests. $\mu$ is the mean value, $\sigma$ the standard deviation.

Data are coherent with those shown in Table 1, taken from the PA's data sheet: flexural modulus and yield stress appear to be slightly smaller that those of pure bulk material, due to the porosity resulting from the sintering process.

\subsection{Digital Image Correlation (DIC)}

Comparison of digital images captured before and during tests is the basis of the DIC method to determine sample's displacement and strain field. This is performed by matching subset of pixels between two images: location of a point in an undeformed subset is found in the deformed image, and thus its displacement is determined. For this study, a 3D Dantec Dynamic Q-400 system equipped with two 5 MP, 17-mm lens, LED illuminated cameras was used. The images were processed by Istra-4D software (Dantec Dynamics). As advised by [16], in order to precisely locate the crack tip, it is important to have accurate measurement of the strain field and high spatial resolution at the same time. In order to achieve that, a facet size of 25 pixels, grid spacing of 11 pixels and contour smoothing of 9 facets was set. The specimen surfaces were prepared with a speckle pattern, as shown in Figure 4, following the procedure prescribed in [16]. 

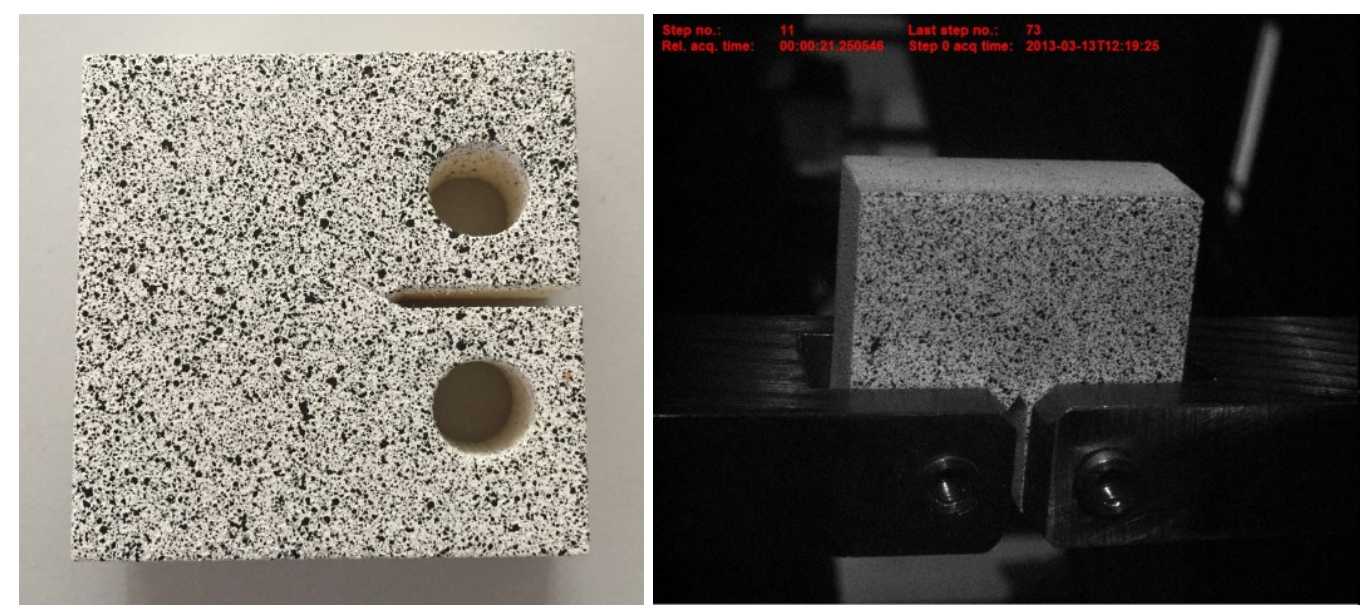

During the test, the maximum strain was measured with the DIC in order to monitor the crack growth.

Moisture absorption was calculated measuring sample's weight before and after 6 days in oven at $80^{\circ} \mathrm{C}$ for 6 days: it is resulted to be $0.363 \%$. Density of the specimens was measured by a Mettler Toledo MS-DNY-54 balance, and found to be equal to $1.00 \mathrm{Kg} / \mathrm{dm}^{3}$.

\section{Results}

\subsection{Mechanical results}

As mentioned above, 3 specimens per configurations have been tested: Figure

3.1 only shows the representative force-displacement curves of each tested configuration for clarity.

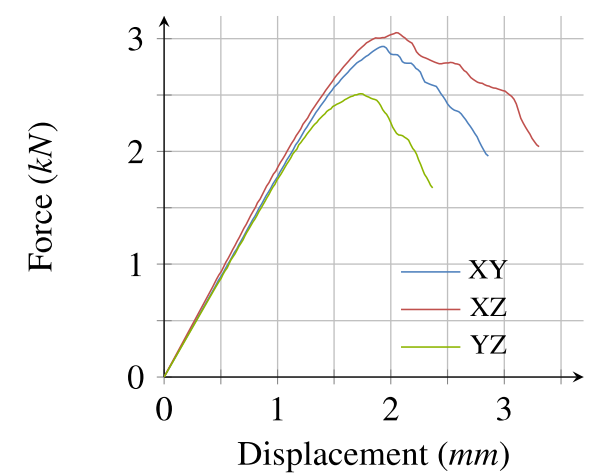

(a) MAN Force-Displacement curves

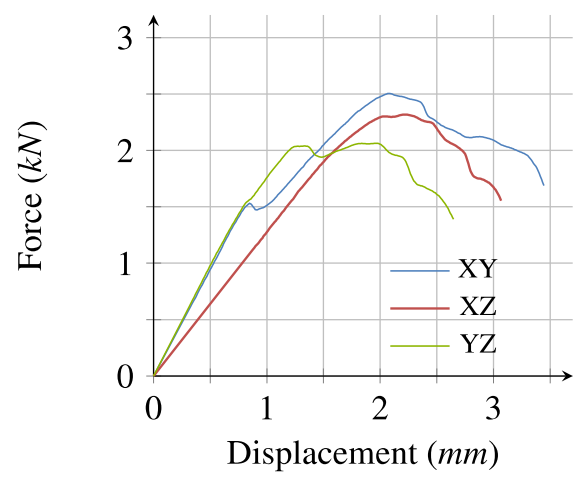

(b) SLS Force-Displacement curves

Due to the above mentioned differences in crack lengths, MAN and SLS forcedisplacement curves are not comparable (specimens with longer cracks are 
more compliant, resulting in lower maximum force); nevertheless, curves within each category can still be compared, and it can be noted that the orientation has a significant effect on both the configurations. Table 3 reports the averaged maximum force measured during the tests.

\begin{tabular}{|c|c|c|c|c|c|}
\hline \multirow{2}{*}{ Configuration } & \multicolumn{5}{|c|}{ Maximum force $(\mathrm{N})$} \\
\hline & $\mu$ & $\sigma$ & & $\mu$ & $\sigma$ \\
\hline XYMAN & 3.03 & 0.07 & $\mathrm{XYSLS}$ & 2.46 & 0.03 \\
\hline $\mathrm{XZ}_{\text {MAN }}$ & 3.04 & 0.14 & XZsLS & 2.34 & 0.01 \\
\hline $\mathrm{YZ}_{\text {MAN }}$ & 2.51 & 0.02 & YZsLS & 2.10 & 0.06 \\
\hline
\end{tabular}

Table 3: Maximum force recorded during tests. $\mu$ is the mean value, $\sigma$ the standard deviation.

Figure 5(b) shows that the curves of XY and YZ sintered specimens follow a bilinear loading phase which is due to anomalous and unwanted gluing of the two surfaces of the crack tip, caused by the resolution of the sintering machine. Those specimens have the crack plane crossed by several layers and, therefore, the laser beam runs close to the crack tip several times. The laser beam has small but not null dimensions, and it locally sintered small portion of the material between the two planes forming the crack, causing partial bonding of the two. Therefore, during the first stage of the tests, the crack length is in truth shorter than expected, and the first load drop is due to breaking of the said pre- gluing. This is an unwanted and unexpected issue that does not, however, influence the calculation of maximum force and the other fracture parameters. Results are reported in Figure 6 and Table 4. 


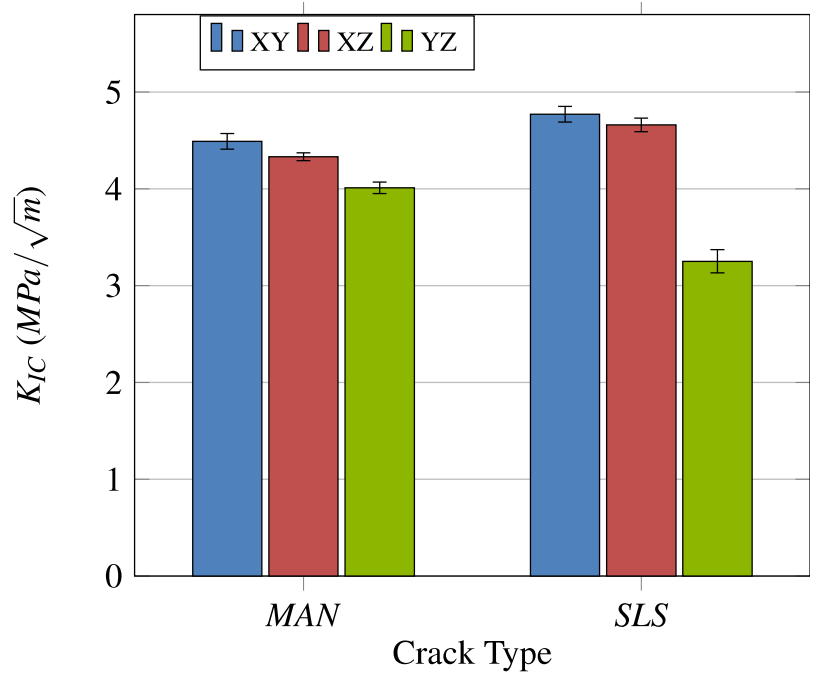

\begin{tabular}{|c|c|c|c|c|c|}
\hline \multirow{2}{*}{ Configuration } & \multicolumn{5}{|c|}{ Stress intensity factor $\left(\mathrm{MPa} / \mathrm{m}^{1 / 2}\right)$} \\
\hline & $\mu$ & $\sigma$ & & $\mu$ & $\sigma$ \\
\hline XYMAN & 4.49 & 0.08 & XYSLS & 4.77 & 0.08 \\
\hline XZMAN & 4.33 & 0.06 & XZsLS & 4.66 & 0.07 \\
\hline YZMAN & 4.01 & 0.04 & YZsLS & 3.25 & 0.12 \\
\hline
\end{tabular}

Table 4: KIC for each configuration. $\mu$ is the mean value, $\sigma$ is standard deviation

\subsection{DIC results}

Figure 7 shows examples of DIC pictures for MAN (Figure 7(a)) and SLS (Figure $7(\mathrm{~b}))$ specimens.

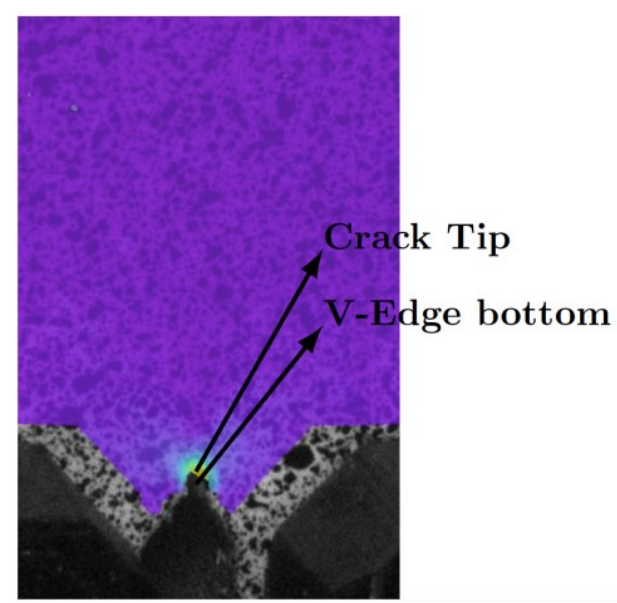

2 mm IAN specimen: crack length and stress concentration

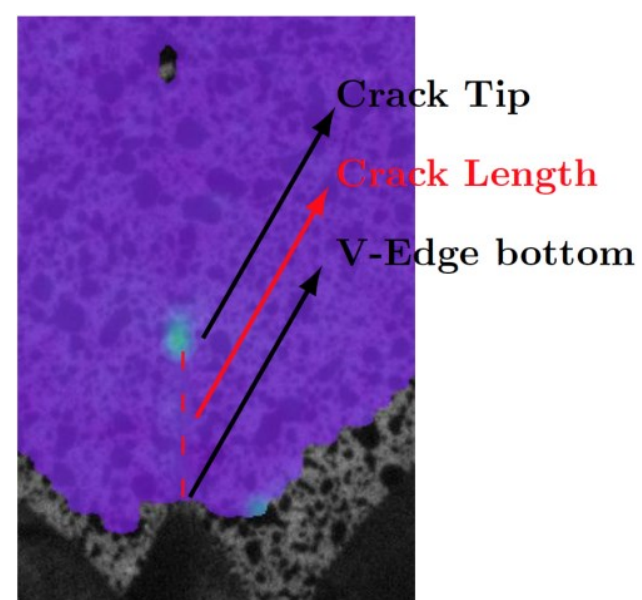

$12 \mathrm{~mm}$ specimen: crack length and stress concentration 
The pictures show the difference in crack length mentioned in $\S 2$ and shown in Figure 3. For both configurations, the maximum deformation and, therefore, the maximum stress, is located at the crack tip: for MAN samples it is much closer to the bottom of the V-edge than the $S L S$ ones.

During the tests, the evolution of the first principal strain field has been monitored, and is presented in Figure 8; the correspondent points are marked on the force vs. displacement graphs on the left. 


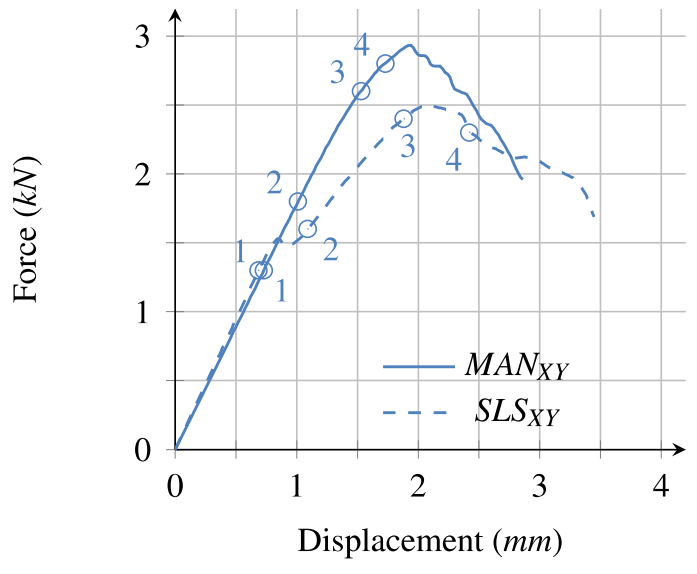

a) XY specimens graphs

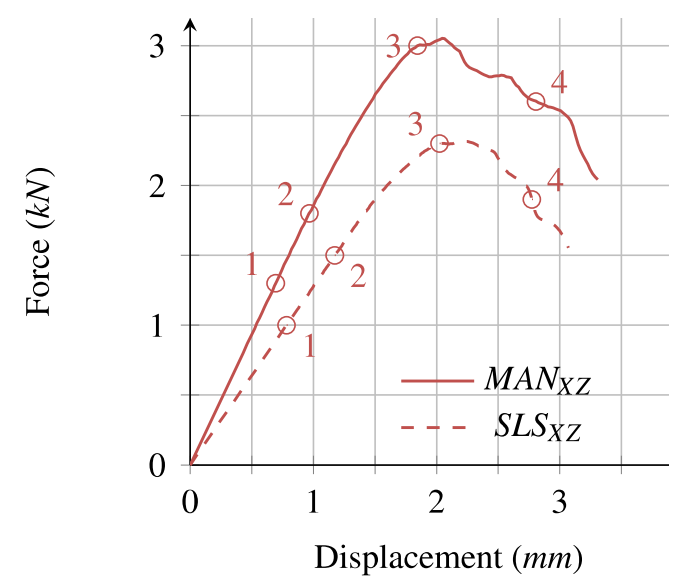

d) XZ specimens graphs

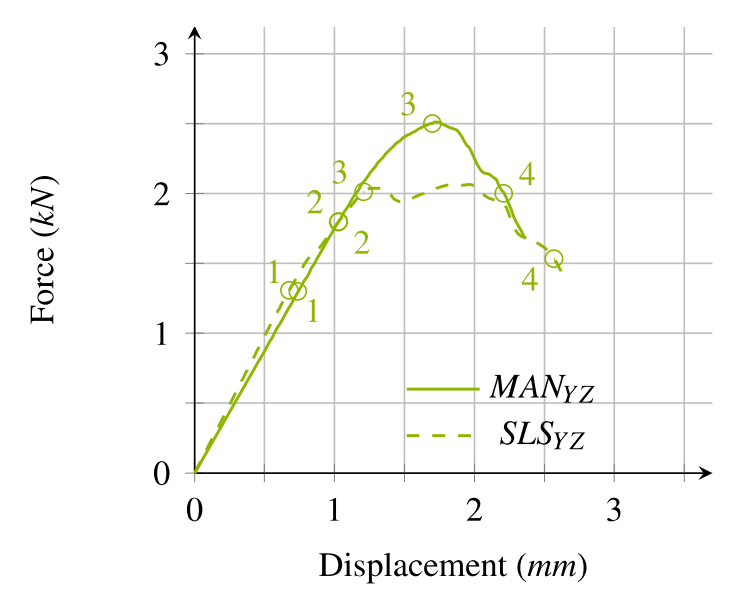

g) YZ specimens graphs

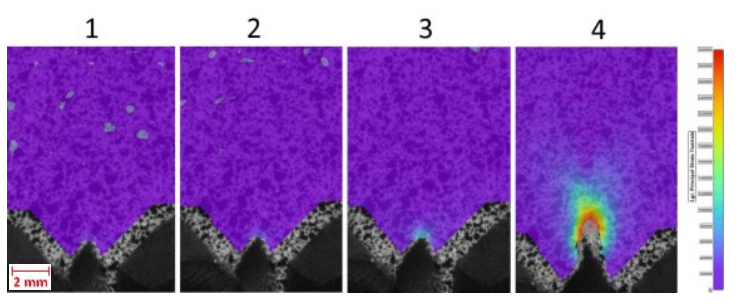

b) XYMAn specimens DIC pictures

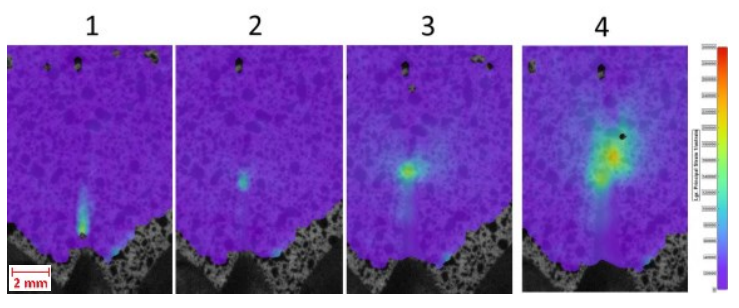

c) XYsLs specimens DIC pictures

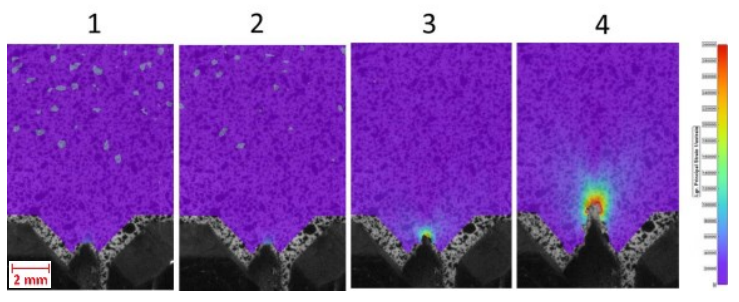

e) XZ $Z_{\text {MAN }}$ specimens DIC pictures

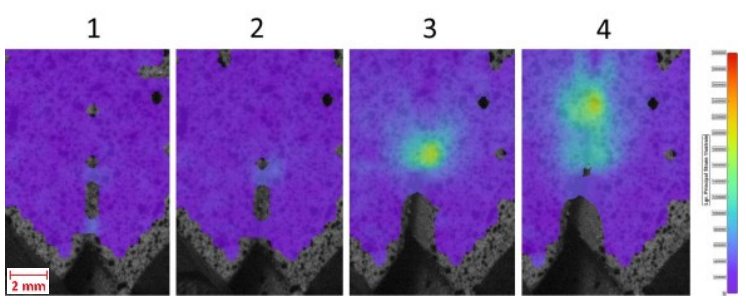

f) XZsts specimens DIC pictures

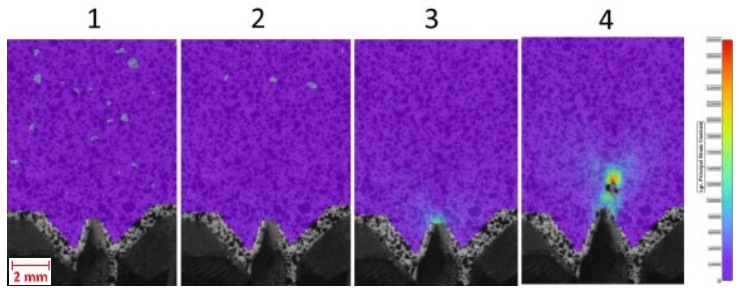

h) YZMAN specimens DIC pictures

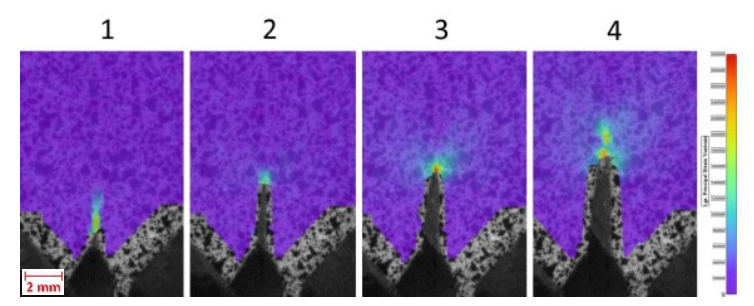

i) YZsLs specimens DIC pictures 
During the linear phase of the tests, the stress concentration around the crack increases, allowing following the propagation of the crack tip.

$M A N$ specimens present a lower stress concentration during the linear phase, as shown in the Image 1: the stress level is so low compared to that in the SLS specimens that it is not even registered. MAN specimens continue having a linear behavior until the peak load (Image 3). Stress concentration is very close to the tip of the V-edge, showing that the crack is not propagating. Once the specimen breaks, the tip of the V-Edge propagates, as so does the crack (Image 4).

SLSxz specimens show a linear behavior until almost the same displacement of the MAN configuration; the lower slope of the curve is due to the longer crack length.

Anomalous behavior has been registered for XYsLS and YZsLS specimens: compared to the $\mathrm{XZ}$ orientation, the slope of the curve is much higher, the stress concentration is closer to the V-Edge, and the load-displacement curves show an unexpected load drop before the maximum load (Images 2). In particular, images of Figure 8(c) show that the stress concentration is close to the V-Edge before the load drop (Image 1) and then quickly move forward where it is supposed to be (Image 2).

Such behavior is due to the pre-gluing of the two surfaces of the crack tip already mentioned in §3.1. At the beginning of the test, a much lower crack is loaded, due to the bonding of the two plane supposed to form the crack, which gives the higher slope of the initial part of the curve. When the material bonding the two planes breaks, then the load drops, and the crack length goes back to normal. As mentioned before, it does not affect the maximum force or its relative strain, as shown by the fact that $\mathrm{XY}$ and $\mathrm{XZ}$ curves reach their maximum at similar points for both the $S L S$ and MAN configurations, and these latter are not affected by any pre-gluing.

\subsection{Fractography}

Figure 9 shows the fracture surfaces for each kind of specimen. 


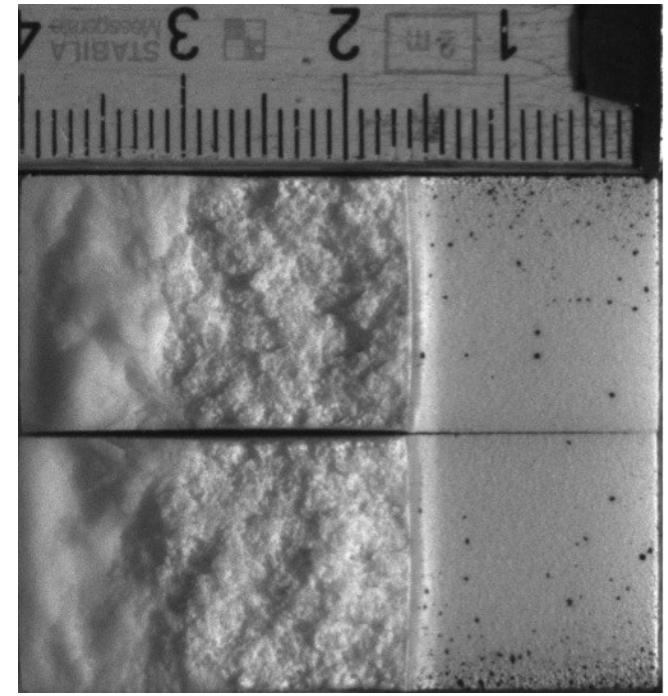

(a) $X Y_{M A N}$ fracture surfaces

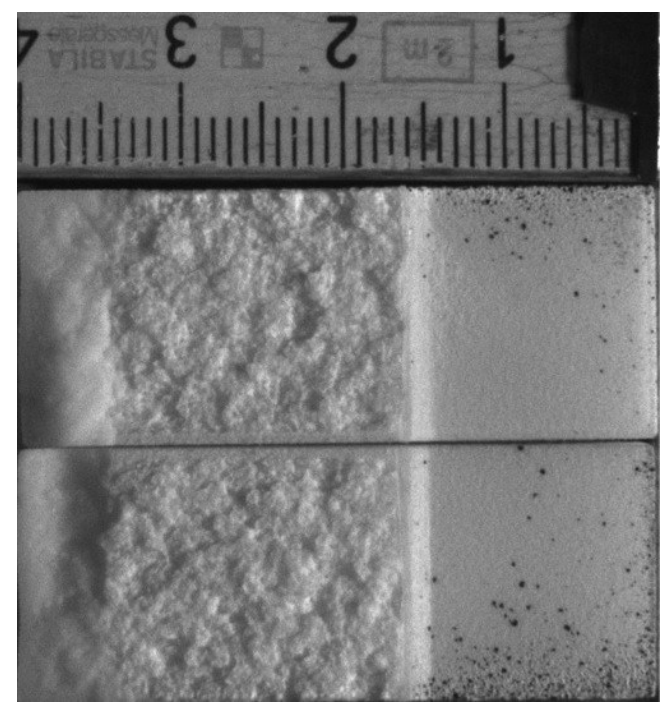

(c) $\mathrm{YZ}_{M A N}$ fracture surfaces

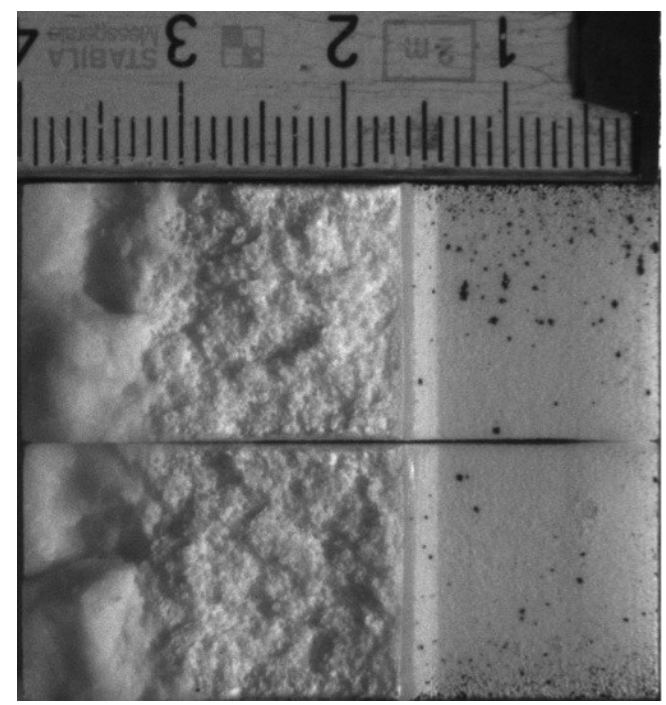

(e) $\mathrm{XZ}_{\text {MAN }}$ fracture surfaces

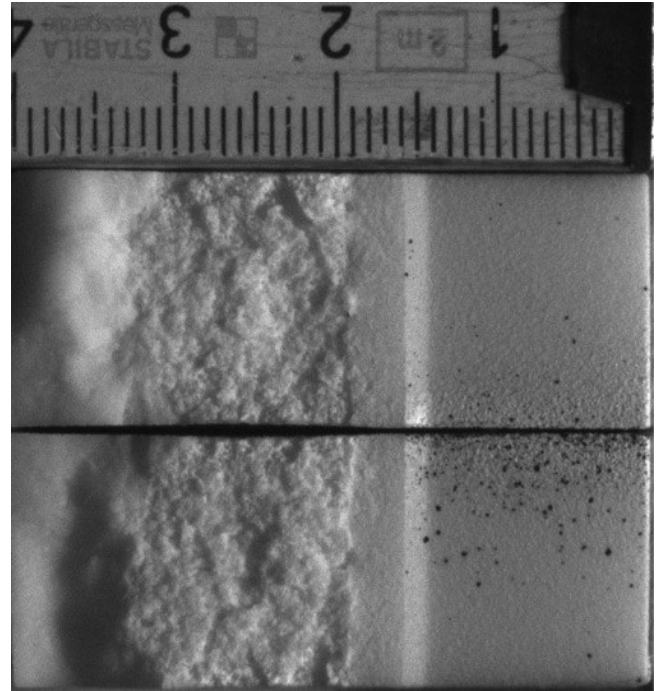

(b) $\mathrm{XY}_{S L S}$ fracture surfaces

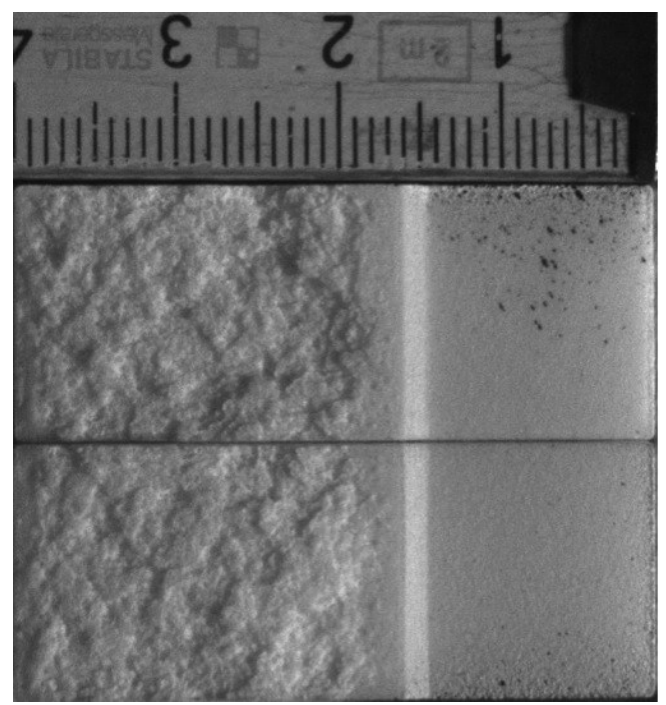

(d) YZsLs fracture surfaces

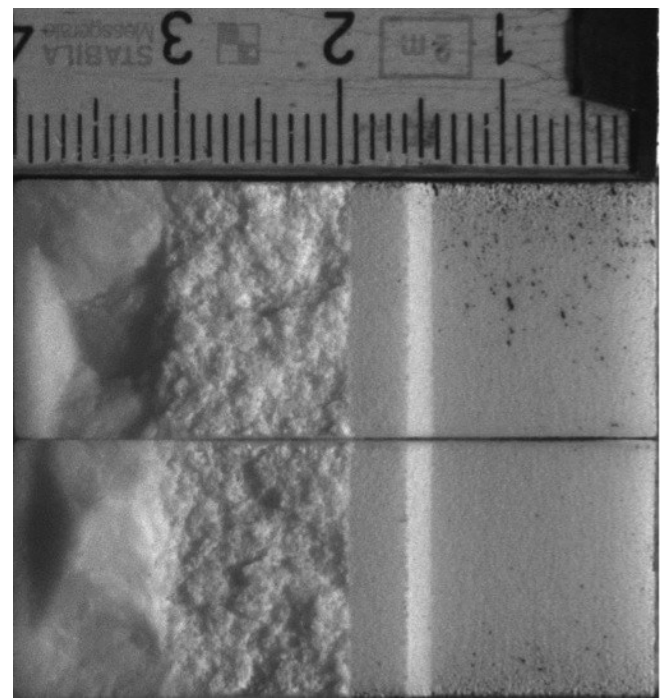

(f) XZsLs fracture surfaces 
Two main differences between $M A N$ and $S L S$ specimens can be observed:

1. fracture surfaces of the YZ specimens (see Figure 9(c) and 9(d)) appear smoother and more flatter than the others, in particular if compared with $S L S$ specimens;

2. in SLSXY and SLSYZ sintered specimens (see Figure 9(b) and 9(d)), the initial crack front is not as sharp and straight as the other configurations, and the surface of the pre crack is not smooth, showing non complete separation of the two crack planes during the manufacturing process.

Pictures clearly indicate a preferential pathway for crack propagation in YZ samples (in the interlayer). On the other hand, the crack paths in XY and XZ samples are longer because geometry forces them to cross other plies, moving in various directions, increasing the energy per unit volume necessary to propagate.

\section{Discussion}

The results provide useful information to understand the effect of the building direction, and the differences between the two crack manufacturing processes.

\subsection{Effect of building direction}

Fracture toughness results were very similar for the two types of crack:

- XY specimens present the highest values of KIC for both SLS and MAN types of specimen;

- XZ specimens register a very small, even though large enough to be detected, reduction of fracture toughness compared with XY specimens. In particular, Table 4 shows that $M A N$ and $S L S$ samples register a reduction of $3.5 \pm 2 \%$ and $2.3 \pm 2 \%$ respectively, compared to the $\mathrm{XY}$ orientation;

- YZ specimens have the weakest fracture toughness, presenting a sharp drop when compared with the other two configurations. In particular Table 4 shows that reductions of $7.5 \pm 1 \%$ and $30 \pm 3 \%$ is registered for $M A N$ and SLS specimens, respectively, compared to XY orientation. As expected, results indicate that the interlayers are the weakest part of the specimen, creating a preferential and easy direction for the crack to propagate. It 
is also confirmed by the fact that fracture surface of the $\mathrm{YZ}$ specimens is much smoother and more planar than the other two configurations, suggesting the presence of a preferential path with lower toughness for the crack to propagate.

\subsection{Type of crack}

Table 4 and Figure 6 show that KIC of XY and XZ orientation of SLS specimens presents an increment of $6 \%$ and $7 \%$, respectively, compared to the same lay-up of specimens in which the crack is manually initiated. This is due to the fact that crack tips manufactured via rapid prototyping are not as sharp as those manually created, inducing lower stress concentration and resulting in higher KIC.

Focusing on the YZ samples, which present a much lower Kic than the other orientations, Figure 6 shows that the SLS specimens are weaker than the MAN ones. This is due to the fact that the $S L S$ crack, initiated during the manufacturing process, lays exactly between two consecutive layers and is, therefore, able to quickly propagate in the preferential direction (the interlayer). On the other hand, the cracks in MAN specimens initiate from a random point and the probability of starting the propagation exactly at the interlayer is lower than the relative $S L S$ cracks. This aspect is confirmed by the fracture surface of YZsLS specimen that appears flatter than the YZMAN .

Therefore, the KIC calculated for the two different manufacturing techniques of $\mathrm{XY}$ and $\mathrm{XZ}$ orientation is similar and comparable; the same does not apply to $\mathrm{YZ}$ configuration.

This result may be of particular interest for further development in fracture mechanics. The close results obtained for MAN and SLS configurations would allow researchers to investigate fracture mechanics by manufacturing components, via AM, with cracks already embedded in the sample. This will bring two main advantages:

- no need for manual crack initiation, either with razor blades or fatigue propagation, improving reliability and standardization of samples and results, and reducing time and costs of the experiments;

- Introduce the possibility to create cracks otherwise impossible with standard techniques. For example it would be possible to create 
components with internal cracks, or with cracks too small to be otherwise manually initiated, or with cracks of different geometries, or cracks in non-accessible positions. It would improve the study of fracture mechanics, for general and specific purposes. For example, one could test real components including internal defect to simulate possible failure of material directly on the geometry of the final component.

\section{Conclusions}

The results of an experimental investigation on SLS 3D printed specimens are presented. The aim of the paper is to assess critical issues on crack propagation focusing on building direction and type of crack. Six configurations have been manufactured considering three building directions and two types of crack. Main results indicate that:

- samples which all the layers contain a portion of the crack results in higher maximum force and fracture toughness;

- samples which layers are parallel to the crack plan offer a preferential path- way for the crack to propagate, resulting in low resistance and toughness;

- while designing components for SLS it is important to avoid geometries that may cause unwanted pre-gluing of sharp edges, which may occur when a narrow edge is crossed by several layers;

- SLS notched specimens show a slight higher KIC compared to the MAN ones due to a less sharp crack tip. However, the difference between the two configurations is small, and the three orientations have the same behavior.

The results offer important indications when designing for 3D printing but also are a good starting point for further studies in the field of fracture mechanics. In particular, the coherent KI C behavior of $M A N$ and $S L S$ specimens allows researchers to use SLS to manufacture samples with internal cracks, and/or with crack at various orientation to study fracture mechanics of complex 3D samples otherwise impossible with traditional techniques. 


\section{References}

1. S. Kumar. Selective laser sintering: a qualitative and objective approach. Jom, 2013;55(10):43-47.

2. L.M. Sherman. Additive Manufacturing: Materials for "Real-World" Parts. Plast Technol, 2014;60(3):42-47.

3. J.P. Kruth, P. Mercelis, J. Van Vaerenbergh, L. Froyen, and M. Rombouts. Binding mechanisms in selective laser sintering and selective laser melting. Rapid Prototyping J, 2005;11(1):26-36.

4. W. Cooke, R.A. Tomlinson, R. Burguete, D. Johns, G. Vanard. Anisotropy, homogeneity and ageing in an SLS polymer. Rapid Prototyping J, 2011;17(4):269-279.

5. G. Berti, L. D’Angelo, A. Gatto, L. Iuliano. Mechanical characterisation of PAAl203 composites obtained by selective laser sintering. Rapid Prototyping J, 2010;16(2):124-129.

6. W. Zhang, R. Melcher, N. Travitzky, R.K. Bordia, P. Greil. Three- Dimensional Printing of Complex-Shaped Alumina/Glass Composites. Adv Eng Mater, 2009;11(12):1039-1043.

7. Y. Shi, J. Chen, Y. Wang, Z. Li, S. Huang. Study of the selective laser sintering of polycarbonate and postprocess for parts reinforcement. P I Mech Eng L-J Mat, 2007;221(1):37-42.

8. V. Cain, L. Thijs, J. Van Humbeeck, B. Van Hooreweder, R. Knutsen. Crack propagation and fracture toughness of Ti6Al4V alloy produced by selective laser melting. Add Man, 2015;5:68-76.

9. B. Vrancken, V. Cain, R. Knutsen, J. Van Humbeeck. Residual stress via the contour method in compact tension specimens produced via selective laser melting. Scripta Mater, 2014;87:29-32.

10. W.H. Peters W.F. Ranson. Digital imaging techniques in experimental stress analysis. Opt Eng, 1982;21(3):427-431.

11. S.R. McNeill, W.H. Peters, M.A. Sutton. Estimation of stress intensity factor by digital image correlation. Eng Fract Mech, 1987;28(1):101-112.

12. ISO (2003). ISO 13586. Plastics - Determination of fracture toughness (GIC and KIC) - Linear Elastic Fracture Mechanics (LEFM) approach.. 
13. D.J. Hitt, B. Haworth, N. Hopkinson. Fracture mechanics approach to compare laser sintered parts and injection mouldings of nylon-12. P I Mech Eng B-J Eng, 2011;225(9):1663-1672.

14. T.L. Anderson. Fracture Mechanics. 2005.

15. M. Cayard. Fracture Toughness Testing of Polymeric Materials. PhD thesis, Texas A\&M University, 1990.

16. M. Palanca, T.M. Brugo, L. Cristofolini. Use of Digital Image Correlation To Investigate the Biomechanics of the Vertebra. J Mech Med Biol, 2015;15(2):1540004-1-10. 